Annuaire suisse de politique de développement

16 | 1997

Environnement et développement, 5 ans après Rio

\title{
La coopération entre Madagascar et la Suisse : la dimension environnementale
}

\section{Michèle Rajaonarivony}

\section{OpenEdition}

1 Journals

Édition électronique

URL : http://journals.openedition.org/aspd/816

DOI : 10.4000/aspd.816

ISSN : 1663-9669

Éditeur

Institut de hautes études internationales et du développement

\section{Édition imprimée}

Date de publication : 1 mars 1997

Pagination : 255-270

ISSN : 1660-5934

\section{Référence électronique}

Michèle Rajaonarivony, «La coopération entre Madagascar et la Suisse : la dimension

environnementale », Annuaire suisse de politique de développement [En ligne], 16 | 1997, mis en ligne le 08 août 2012, consulté le 08 septembre 2020. URL : http://journals.openedition.org/aspd/816 ; DOI : https://doi.org/10.4000/aspd.816 


\title{
LA COOPÉRATION ENTRE MADAGASCAR ET LA SUISSE : LA DIMENSION ENVIRONNEMENTALE*
}

\author{
Michèle RAJAONARIVONY
}

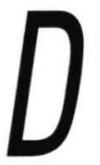

ans un contexte mondial de course à la croissance et de démographie galopante, l'entrée des pays les moins avancés dans le troisième millénaire semble bien difficile. La dégradation de l'environnement résultant de la surexploitation des ressources, liée à la paupérisation des populations de ces pays, est forcément devenue une préoccupation majeure pour les agences de coopération au développement. Désormais, ces dernières se donnent pour mission d'accompagner leurs partenaires sur la voie délicate de l'apprentissage d'une gestion plus durable de leurs ressources naturelles. Dans cette perspective, la Direction pour le Développement et la Coopération (DDC) a tenté de renforcer la dimension environnementale dans son programme de coopération avec Madagascar.

Après une mise en situation dans le paysage malgache et une présentation des spécificités de la problématique environnementale dans la Grande Ile, nous tenterons d'expliciter les approches mises en æuvre dans le programme de coopération soutenu par la DDC. L'accent sera mis sur les différents enjeux et sur les apprentissages réalisés. Une discussion des succès et des échecs rencontrés permettra de brosser un bilan synthétique de cet effort pour mener une coopération qui place l'environnement, espace de vie de toute une population, au centre des préoccupations.

\section{UNE JOURNÉE SUR LES HAUTS-PLATEAUX MALGACHES}

Ce bel après-midi de dimanche, un petit hameau de quinze maisons de campagne se prépare à la fête. Une brise légère dévoile leurs façades rouge brique de dessous les figuiers centenaires qui les abritent. Quelques fumées éparses s'échappent encore des typiques toits de chaume mais, aux premiers échos de tambour, les occupants ont quitté leur cuisine pour se rendre au lieu du rendez-vous. Ce terrain vague, qui d'ordinaire fait office de terrain de football, est déjà noir de monde. De nombreuses familles sont installées là, en tailleur ou accroupies sur l'herbe sèche et rase. Couvertes d'un chapeau de paille ou d'une étoffe, les têtes bougent dans tous les sens, qui pour guetter la venue des retardataires, qui pour admirer les artistes. Dans tous les cas, petits et grands commentent déjà les mouvements d'échauffement du plus jeune danseur de la troupe. Et, lorsqu'ils sentiront avoir réuni les meilleures conditions de diction, de prestance et d'attention, les mpihira gasy taperont des pieds et des mains, au rythme de la grosse caisse, pour marquer le début du spectacle. En avant la musique...

\footnotetext{
* Mes plus vifs remerciements vont à M. Kuno Schläfli (chargé de programme Madagascar à la Centrale de la DDCde 1993 à 1996) et à M. Philippe Zahner (coordinateur de la DDC à Madagascar de 1991 à 1996) qui ont bien voulu commenter à mon intention la littérature consultée et répondre à mes nombreuses questions. Ma gratitude leur est en outre acquise pour avoir accepté de revoir le texte.
} 
Les mpihira gasy sont aux Malgaches ce que furent un jour les troubadours aux Européens. Ils sillonnent les campagnes et interprètent des airs traditionnels dont ils ont actualisé les textes. Les spectacles se présentent sous forme d'échanges oratoires entre deux parties : d'un côté les hommes et, de l'autre, les femmes. Les premiers critiquent, les secondes répliquent, et vice versa. Chaque fois, les deux dénoncent des situations compromettantes dont les uns et les autres essaient de se justifier. Drapés de costumes bariolés et virevoltants, ils déclament des scènes de vie quotidienne où l'humour véhicule des messages moralisateurs. Ils enchâ̂nent des acrobaties compliquées de leurs pieds nus et agiles. Les uns chantent, les autres dansent et l'ensemble fascine par sa popularité.

Nouveauté du jour : le thème choisi par nos mpihira gasy s'est écarté de la corruption ou de la jalousie pour traiter de sujets plus terre à terre. « Les arbres qui entourent les villageois, les champs qu'ils labourent, les collines que leur bétail traverse, l'eau qu'ils puisent ; bref, leur environnement et eux-mêmes sont en danger » répète, telle une litanie, le texte des chansons. Pour la première fois aussi, les paysans apprennent qu'ils sont en mesure de moins s'endetter en diversifiant leurs cultures ou qu'ils peuvent économiser de l'eau en améliorant leur système d'irrigation. Leur attention se meut en intérêt, toutefois mêlé de scepticisme. Plus que des messages en chansons, ils préféreraient des propositions concrètes à leurs problèmes environnementaux. Car bouleverser des pratiques qui ont fait leur preuve des générations durant ne leur vient pas spontanément à l'esprit. Devoir les remplacer paraît encore moins évident. Et quand les mises en garde proviennent de chanteurs d'origine paysanne, comme eux, la perplexité est à son comble... Au milieu de toutes ces interrogations, les villageois ont toutefois remarqué une chose : les chanteurs sont accompagnés d'une petite équipe de personnes en tenue de ville. Et la présence de ces dernières n'est pas que de la figuration : de l'argent, du matériel et des techniques doivent être en jeu. « Mais pourquoi donc via les mpihira gasy?»

En fait, la Coopération suisse et le WWF soutiennent ces artistes, car ils parviennent à toucher le coeur des villageois, là où nombre de techniciens et de conseillers ruraux ont échoué. Pour expliquer comment les familles trop nombreuses mettent en danger les forêts, participent à la stérilisation des sols et à la disparition d'espèces endémiques, ils utilisent un langage simple, direct, compréhensible par tous. Un langage qui, sans être accusateur, décrit sans complaisance la situation dans laquelle vivent les paysans et met le doigt sur leur responsabilité par rapport à cette situation. En choisissant le canal culturel pour parler d'environnement, la Coopération suisse et le WWF, par le biais de ces danseurs folkloriques, touchent le Malgache en son point le plus réceptif : le plaisir des joutes oratoires et du langage parlé, la force du discours traditionnel (kabary).

Cette approche du développement rural par la dimension culturelle - dont l'exemple des mpihira gasy - ne représente d'ailleurs qu'une option parmi les six que Madagascar et la Suisse tentent de mettre en œuvre pour s'attaquer à la problématique environnementale dans leur programme de coopération pour les années 1995-2000. Cet éventail de six approches est le résultat de plus de deux décennies d'apprentissage, notamment dans le domaine du développement rural, et s'inscrit dans la perspective des résolutions prises au sommet mondial de Rio de Janeiro en 1992. Mais avant d'entrer dans la discussion de ces approches mal- 
gacho-suisses - et surtout des résultats qu'elles ont enregistrés -, replaçons brièvement nos spectateurs de mpihira gasy dans leur environnement global : Madagascar et ses particularités.

\section{L'ENVIRONNEMENT À MADAGASCAR : QUELS SONT LES ENJEUX ?}

La « révolution verte » n'a pas eu lieu à Madagascar. D'ailleurs, l'expression est dépassée depuis que les notions de « développement durable » et d' "équité dans le partage des bénéfices de la croissance » résonnent dans toutes les oreilles. La Grande Ile est riche d'un potentiel énorme que le monde entier lui reconnaît. Grande comme 14 fois la Suisse (France et Bénélux réunis), elle abrite une flore et une faune endémiques à $85 \%$. Bon nombre de reptiles, de poissons et d'orchidées, sans oublier la plupart des espèces de lémuriens et de baobabs qui y vivent, ne se retrouvent nulle part ailleurs. Elle offre non seulement une nature généreuse, mais surtout des spécimens médicinaux prouvés ou en puissance, des merveilles géologiques et marines uniques, une diversité de cultures encore empreintes de toutes leurs traditions.

Les Hauts-Plateaux malgaches se distinguent, du point de vue géologique, par leurs sols latéritiques où pousse une végétation généralement pauvre, à l'exception des sols volcaniques de l'ouest et du sud. Les collines (tanety) usées par l'érosion, striées de lavaka (sols effondrés sous l'effet du ruissellement), clairsemées de bosquets d'eucalyptus et de pins, sont régulièrement éprouvées par les feux de brousse. Ces Hauts-Plateaux satisfont néanmoins la majeure partie des besoins en produits vivriers et maraîchers de la capitale et de plusieurs autres grandes villes, y compris sur la côte est du pays. Tout le reste du territoire qui descend sur les plaines côtières se présente sous forme de sédiments sablonneux, rocheux ou calcaires. Mais pour peu fertiles qu'ils soient, ces sols abritent les 12 réserves naturelles intégrales de l'île (totalisant près de 600000 hectares), un de ses deux parcs nationaux et la majorité des réserves spéciales (une vingtaine).

\section{Madagascar en bref 1}

Population de 13.4 millions d'habitants en 1992 (dernier recensement national)

Environ $25 \%$ de cette population vit en ville

Croissance démographique de $3.2 \%$ par an

$81 \%$ de la population active dans l'agriculture

Espérance de vie à la naissance de 56.5 ans

8'333 personnes par médecin

$1.3 \%$ du PIB pour la santé

$2.3 \%$ du PIB pour l'enseignement primaire

Superficie de 582 ' $000 \mathrm{~km} 2$ dont $5.3 \%$ de terres arables et $26.6 \%$ de terres boisées

Taux annuel de déboisement de $1.2 \%$ par an

1 Gouvernement de Madagascar, Ministère chargé du Plan, 1992, 1993, 1994. 
PNB total de 2.8 milliards de US\$ dont :

PNB par habitant de 230 US\$

Production agricole qui représente $33 \%$ du PIB

Production industrielle qui représente $14 \%$ du PIB

Dette extérieure totale de 4.4 milliards de US\$

Service de la dette qui revient à $19 \%$ des exportations

Exportations égalant $63 \%$ des importations

Aide publique internationale :

- environ 350 millions de US\$ en 1994, dont $29 \%$ dans le secteur productif, $45 \%$ dans les infrastructures, $18 \%$ dans le secteur social et le reste dans le secteur administratif

- les principales sources sont l'IDA (35\%), la France (24\%), l'Union européenne (15\%), les USA (6\%), la Suisse (4\%) et autres (16\%), dont l'Allemagne, le Japon, etc.

La population de Madagascar reste rurale pour ses quatre cinquièmes. Ainsi, l'économie du pays repose essentiellement sur l'exploitation des ressources naturelles. Et, comme la régénération de ces ressources ne parvient pas à suivre le rythme élevé de la croissance de la population (près de 3\% par an), elles sont gravement menacées de destruction. Il ne reste plus que $27 \%$ de terres boisées et, chaque année, 200'000 hectares supplémentaires de forêts sont rayés de la carte. Au début des années 90 , les terres régulièrement exploitées pour l'agriculture ne représentaient qu'un peu plus du vingtième du territoire, et n'étaient irrigables que pour un tiers. En conséquence, la population malgache s'appauvrit toujours davantage. Actuellement, la plus grande partie vit en dessous du seuil de pauvreté : quatre personnes sur cinq en milieu urbain et trois sur cinq en milieu rural. La situation est donc plus que préoccupante. D'ailleurs, un récent rapport ${ }^{2} \mathrm{du}$ secrétariat du Fonds mondial pour l'environnement (FME) reconnaît à Madagascar un caractère « unique en matière de biodiversité dans le monde » et déclare qu'il s'agit de le «sauvegarder prioritairement, de par sa haute richesse, son endémisme et le degré de menace qu'il encourt ».

Face à cette situation, la communauté internationale s'est mobilisée massivement, à l'appel des environnementalistes, pour financer des projets grandioses de sauvegarde de ce cadeau de la nature. L'idée est de supporter le (PAE) national qui a vu le jour en 1989.

\section{Le Plan d'Action Environnemental 3}

Lancé en 1989

Se base sur la Charte Environnementale

Porte sur une durée de 15 ans

Prévu pour être réalisé en phases de 5 ans

Soutenu par un pool de bailleurs de fonds animé par la Banque mondiale :

- Banque mondiale, PNUD, FEM et FAO,

- Allemagne, Etats-Unis, France, Japon, Norvège, Suisse et Union européenne

2 Global Environment Facility, Proposal for supporting Madagascar Environment Programme, unpubl., 1996.

${ }^{3}$ Gouvernement de Madagascar, Charte de l'Environnement, 1990, 84p. 
- création d'un cadre légal

- élaboration de politiques et stratégies

- mise en place des structures d'exécution

Deuxième phase de 1996 à 2001

- consolidation du travail légal

- consolidation des nouvelles structures

- passage des actions-pilote à une implantation plus large des réalisations opérationnelles

- rééquilibrage des priorités

Troisième phase prévue de 2001 à 2006

- poursuite de l'extension des activités opérationnelles

- intégration des stratégies environnementales dans les politiques sectorielles et dans la politique macro-économique du pays

- phasing out des activités d'appui institutionnel

La deuxième phase met l'accent sur :

- la gestion durable des sols et des ressources en eau

- les écosystèmes forestiers à usage multiple

- la gestion des aires protégées et l'écotourisme

- l'environnement marin et côtier

Un budget d'environ 30 millions de US\$ par an, dont 7 millions US\$ de la part du Gouvernement malgache, est requis pour la réalisation.

Ce Plan d'Action, prévu sur 15 ans, est mis en œuvre par trois projets d'une durée de cinq ans, soutenus principalement par les coopérations américaine, française, allemande, suisse et japonaise, ainsi que par la Banque Mondiale, le FME et les agences des Nations Unies.

\section{QUEL RÔLE POUR LA COOPÉRATION MALGACHO-SUISSE ?}

Bien que soutenant clairement cette démarche de la communauté internationale, la Suisse ne la trouve pas suffisante, parce que plus axée sur la conservation des ressources naturelles que sur une utilisation durable de ces mêmes ressources au bénéfice des populations rurales. Alors que beaucoup de partenaires nationaux ou internationaux ont tendance à limiter l'environnement à l'ensemble des réserves naturelles et parcs nationaux, voire à certains massifs forestiers et à certains récifs coraliens, la DDC donne à l'environnement une définition bien plus large, en tant qu'espace de vie d'une population, avec ses ressources naturelles, mais aussi sa structure socio-économique. ${ }^{4}$ En conséquence, la DDC ne considère sa participation au PAE que comme une des 6 approches qu'elle a retenues dans son programme.

\footnotetext{
${ }^{4}$ Direction pour le Développement et la Coopération (DDC), Stratégie environnementale, 1994, 11 p.
} 


\section{Programme de coopération Madagascar-Suisse 5}

Débute dans les années 60 avec un programme de volontaires

Fin 60-début 70, déploiement d'activités dans l'agriculture, la foresterie et l'enseignement technique

Fin 70, élargissement à l'enseignement universitaire (élevage, foresterie) et à la petite industrie

Un bureau de coordination est mis en place en 1977

Début 80, appuis au secteur santé (médicaments essentiels et adductions en eau potable) et dans les infrastructures routières

Fin 80, préoccupation environnementale se concrétisant par la participation au lancement du Plan d'Action Environnemental

Début 90 , concentration sur les domaines d'intervention suivants :

- Production soutenue et respectueuse de l'environnement (programmes de développement rural dans 3 régions choisies, appuis institutionnels en gestion durable des ressources naturelles, appui à la formulation de politiques sectorielles, contribution aux phases I et II du PAE)

- Actions dans le domaine social (pharmacies communautaires et politique de médicaments essentiels, adductions d'eau villageoises et autres réalisations communautaires, communication rurale, dimension culturelle)

- Infrastructures routières (d'abord réhabilitation, puis mise en œuvre de l'entretien routier sur les axes économiquement importants, participation du secteur privé à l'entretien des dessertes agricoles)

- Coopération humanitaire (contribution à des interventions de la communauté internationale suite à des cyclones, actions de Vivre Contre Travail pour la remise en état d'un important réseau hydroagricole)

- Coopération financière avec l'OFAEE (appui à la balance des paiements dans le secteur santé, réforme du secteur financier)

Entre 20 et 25 millions de francs suisses durant la deuxième moitié des années 80 , le budget consacré par l'Administration Fédérale à ce programme se situe maintenant entre 17 et 19 millions de francs suisses par an, coopérations humanitaire et financière comprises.

Remontant à une trentaine d'années, l'intervention suisse a d'abord touché le domaine de l'enseignement technique. Très vite, le développement rural s'est révélé un enjeu majeur de la coopération malgacho-suisse. D'ailleurs, la vocation de la DDC n'est-elle pas d'améliorer les conditions de vie des couches défavorisées de la population, notamment de la population rurale quand celle-ci est majoritaire ? C'est pourquoi le souci de la dégradation de l'environnement est resté présent à tous les niveaux d'intervention.

Dès lors, que des caravanes de musiciens suisses ${ }^{6}$ et malgaches sillonnent la Grande Ile un mois durant en communiquant des messages culturel et environnemental ; que des centaines d'hectares de forêts secondaires de l'ouest du pays soient épargnées en offrant aux villageois des possibilités d'exploiter durable-

5 Sur la base de : DDC, Programme par pays Madagascar 1995-2000, non publié, 1994, 21p. DDC, Programme de coopération Madagascar-Suisse 1994, 1995, 1996.

${ }^{6}$ Les rockers bernois Patent Ochsner et l'accordéoniste vaudois Michel Besson. 
ment les terres restées en jachère ; que des chaînes de collines rasées par les feux de brousse soient remises en valeur sur les Hauts-Plateaux... ; bref, que les projets s'appellent Gasikara Maitso Volo (Madagascar, verte contrée), Action Monka (terres de défriches) ou Lova Soa (précieux héritage), la relation entre la population rurale et son environnement est le défi majeur posé à toutes les actions.

\section{COMMENT MALGACHES ET SUISSES POURRONT-ILS RELEVER CE DÉFI?}

Les Malgaches attendent de la communauté internationale - si elle veut soutenir le redressement économique du pays - qu'elle contribue à doter les nationaux des compétences et du cadre institutionnel nécessaires à leur participation active et efficace à la vie publique. Toute action menée en collaboration avec les coopérations multilatérales ou bilatérales doit ainsi permettre aux Malgaches d'acquérir davantage d'autonomie, tant économique qu'organisationnelle.

La Coopération suisse, quant à elle, s'inscrit clairement dans cette ligne, tout en optant pour un développement durable (donc respectueux de l'environnement) comme support de son intervention. ${ }^{7}$ La raison de ce choix est triple. D'une part, l'environnement figure parmi les cinq priorités de l'image directrice ${ }^{8}$ de la DDC : « appuyer la population à maîtriser son environnement » est tout aussi important que « lui permettre d'atteindre une certaine autonomie économique », « soutenir les pauvres », « améliorer les ressources de production» et «valoriser les ressources humaines ». D'autre part, la Coopération suisse a pour règle de concentrer ses efforts dans les domaines où elle a rassemblé un riche bagage d'idées et d'expériences, par rapport à un certain contexte national. Ce qui est le cas pour le développement rural à Madagascar. Enfin, cette démarche correspond aux engagements pris par la Suisse lors du sommet mondial de Rio de Janeiro.

En 1996, quatre ans après ce sommet, il est tentant d'analyser l'impact de la démarche helvétique dans le paysage environnemental malgache. Par quels moyens la Coopération suisse répond-elle à sa mission complexe qui est de partager un peu des fruits de sa prospérité avec un pays en développement comme Madagascar et de soutenir la population malgache à tirer le meilleur parti de conditions-cadres très difficiles ?

Chacune des six approches différentes décrites ci-après vise à aborder la problématique sous un angle différent, avec les enjeux et difficultés qui lui sont liés. La variété dans les approches pourrait faire croire que la coopération suisse procède par tâtonnement avant d'opter définitivement pour telle ou telle approche suivant les résultats qu'elle procure. Il faut plutôt voir ici la volonté d'appréhender la problématique de l'environnement sur tous les fronts et - partant - à en faire la base des interventions. Nous tenterons donc d'illustrer dans les paragraphes suivants comment chacune de ces six approches a été mise en pratique, quels résultats ont été atteints et quels problèmes rencontrés.

\footnotetext{
${ }^{7}$ Conseil fédéral, Lignes directrices Nord-Sud Rapport du Conseil Fédéral sur les relations Nord-Sud de la Suisse dans les années 90, 1995, $28 \mathrm{p}$.

8 Direction pour le Développement et la Coopération (DDC), Image directrice, 1991, 89 p.
} 


\section{$\square$ Mieux tenir compte des besoins des paysans dans les actions de développement rural}

La première approche est relative au choix de la DDC de « désectorialiser » ses actions forestières ou agricoles pour les faire tendre vers des programmes de développement rural avec une forte composante de "gestion durable des ressources naturelles ». Pendant les 20 premières années de sa présence à Madagascar, les interventions de la Suisse ont eu trait à divers sous-secteurs de la production agricole, forestière ou zootechnique, soit respectivement un encadrement technique aux institutions de formation professionnelle, de recherche agricole ou de gestion des eaux et forêts, et un appui également technique aux villageois dans le cadre de reboisements, de cultures sèches sur collines ou d'amélioration des méthodes de culture du riz. Peu à peu, cette forme de coopération s'est heurtée à plusieurs limites : les bénéficiaires présupposés de son assistance reçoivent parfois les nouveaux enseignements sans comprendre les raisons de bouleverser les habitudes ancestrales. Les résultats escomptés n'apparaissent pas. Du coup, une démarche différente qui se fonde sur les acquis culturels, enracinés et incontournables, est adoptée. Parole est donnée à la population locale afin qu'elle identifie et exprime elle-même ses besoins. Dès lors, les coopérants sont davantage considérés comme des partenaires et non plus comme des étrangers venus imposer leur savoir-faire en méconnaissant la tradition. Mieux, leur assistance vient suppléer au désengagement progressif du service public tenaillé par un manque de moyens et une désorganisation flagrante. Enfin, face aux préoccupations des villageois touchant l'ensemble de leur terroir, la coopération a reconnu qu'apporter des réponses trop pointues et trop techniques était insuffisant. Il s'est avéré indispensable de considérer la forêt et les terres agricoles comme des parties intégrantes de l'ensemble des ressources naturelles à exploiter de façon durable.

Ouvrir le dialogue avec les villageois sur la base de petites réalisations d'un intérêt commun évident (reboisement d'une parcelle communale, mise en place d'un système de crédit en semences, restauration d'un petit barrage pour l'irrigation), mener avec eux des diagnostics permettant à tous les groupes sociaux de s'exprimer, entrer dans une relation contractuelle fixant aussi bien les objectifs communs que les obligations réciproques, deviennent les étapes usuelles des processus de coopération. De manière générale, l'intérêt des bénéficiaires s'accroît mais en même temps, tout prend plus de temps. La Participation avec un grand $\mathrm{P}$ démontre qu'elle a ses exigences... et les résultats ne se font pas sentir aussitôt. D'abord, cette nouvelle approche dévoile de nombreux conflits d'intérêts. Citons par exemple, dans le cadre de l'appui à la production vivrière sur les Hauts-Plateaux, la réticence des propriétaires des rizières ou des champs à laisser les paysans diversifier par eux-mêmes les cultures et à en tirer équitablement les revenus qui leur sont dûs. Ces propriétaires, du reste souvent citadins, concèdent bien leurs terres à l'exploitation mais d'une part exigent que les trois quarts de la production leur reviennent et, d'autre part, considèrent que le quart restant équivaut autant à une rémunération qu'à un privilège accordé aux travailleurs. Du coup, même si ces derniers voient en la diversification des cultures une possibilité alléchante d'augmenter leurs revenus, les conflits que risque d'engendrer leur désir d'autonomie les font beaucoup hésiter.

Que la coopération s'engage pour la résolution constructive de tels conflits d'intérêts par rapport à la maîtrise des ressources naturelles (et donc des principaux outils de production) est innovateur, positif en soi, mais requiert donc davantage 
de patience, de doigté et une continuelle remise en cause des acteurs de développement. Faire évoluer les mécanismes du pouvoir est porteur d'un véritable développement, mais peut aussi aboutir à une rupture.

\section{$\square$ Participer au débat sur les politiques de développement}

La deuxième approche se situe au plan du débat des politiques de développement, notamment dans le cadre de la mise en œuvre du Plan d'Action Environnemental national (PAE, voir ci-dessus). La contribution suisse au PAE sur le terrain se traduit par un appui stratégique et opérationnel à l'Association Nationale des Actions Environnementales (ANAE) désignée pour accompagner les groupements de paysans dans leur effort pour produire tout en respectant la fragilité de l'environnement.

Au niveau des conditions-cadres, la Suisse a fortement appuyé un processus de redéfinition de la politique forestière malgache et retient parmi ses engagements celui de veiller à sa mise en œuvre progressive. Sur ce plan, les problèmes rencontrés par la Suisse tiennent moins de ses relations avec les bénéficiaires qu'avec les autres bailleurs de fonds et les opérateurs nationaux. Généralement très présents aux côtés des paysans, les coopérants et experts nationaux engagés par la DDC s'appliquent à avoir une perception précise et une «façon de faire » pragmatique. Cette proximité particulière prédispose la Coopération suisse à faire valoir ses expériences auprès des gros bailleurs de fonds qui touchent moins directement les villageois mais qui soutiennent avant tout des structures intermédiaires, étatiques ou non gouvernementales, notamment pour la gestion des aires protégées. Pour prendre l'exemple de la nouvelle politique forestière, l'appui helvétique a essentiellement été justifié par le réalisme de la politique en question. L'Etat n'a plus les moyens qui étaient les siens lors de la période coloniale. Prioriser les champs d'activités, déléguer au secteur privé ce qui peut l'être, renforcer son rôle normatif primordial, telles sont les nouvelles attributions de la Direction des Eaux \& Forêts.

Du côté national, les décideurs politiques sont restés longtemps tiraillés entre le désir d'opter pour une politique plus ambitieuse - et donc de bénéficier d'une enveloppe financière plus importante à gérer - et la volonté de disposer d'un encadrement plus proche des villageois. En fin de compte, ils ont opté pour la seconde voie. Aujourd'hui, bien que le budget qu'elle alloue à l'environnement soit bien plus modeste que celui des coopérations française ou américaine, la DDC dispose d'une expérience qui devrait lui permettre de participer plus activement aux débats sur l'environnement pour défendre les intérêts des ruraux.

\section{$\square$ Introduire l'environnement dans l'éducation nationale}

Une troisième approche s'oriente vers l'éducation primaire : la DDC expérimente une branche originale nommée Sekoly Maitso (Ecole verte). L'environnement comme cadre et source de vie paysanne fait l'objet d'une discipline supplémentaire dans le programme scolaire. L'idée est d'introduire le thème du respect de l'environnement auprès d'enfants scolarisés de 7 à 10 ans. La mise en terre de plantules d'arbres fruitiers et ornementaux, les soins à y apporter, le plaisir de les voir croître deviennent une activité scolaire au même titre que l'histoire ou l'arithmétique. Des pépinières protégées par leurs soins constituent les terrains pratiques des enfants dans leur cour d'école. Puis ils transféreront leur plante dans une petite parcelle qui leur est propre, chez eux. Les enfants non sco- 
larisés, quant à eux, peuvent participer aux fêtes scolaires où sont également commercialisés les petits arbres. Les recettes de telles ventes sont par la suite versées au profit des classes soit pour l'achat de fournitures scolaires, soit pour de petites réhabilitations d'infrastructures (par exemple remettre en état le toit d'une salle de classe endommagé).

L'action-pilote Sekoly Maitso ne touche pour l'instant qu'une cinquantaine d'écoles publiques dans les campagnes avoisinant la capitale malgache. Mais, étant donné l'originalité et l'efficacité du projet, il faut espérer que le principe s'étendra à une plus grande échelle. D'ailleurs, après la première phase de recherche-action, elle s'est vu injecter des crédits supplémentaires pour sa continuité. Une toute petite équipe soutient actuellement cette nouvelle forme de sensibilisation - réussie - des enfants ruraux par le biais de l'école. Quatre personnes couvrent difficilement cinq poches villageoises à $50 \mathrm{~km}$ de la capitale malgache. De plus, les hameaux eux-mêmes se trouvent à plus de $10 \mathrm{~km}$ à l'intérieur des campagnes ; là où, certes, les villageois sont enracinés dans leur campagne profonde, mais aussi là où ils sont le plus difficilement en contact avec d'autres à qui ils pourraient transmettre leurs expériences en un temps relativement court. Une visée régionale ou nationale de cette approche a donc pour l'instant très peu de chances de se dessiner, parce que trop exigeante au plan des moyens (surtout le temps). Pourtant, cette approche, qui implique directement et de la manière la plus précoce les enfants ruraux, est doublement avantageuse pour la population rurale. D'une part, elle inculque aux enfants le respect de leur environnement immédiat au moment où ils manifestent le plus de curiosité pour le monde qui les entoure. De l'autre, elle encourage les familles à l'éducation, car les enfants acquièrent à l'école des expériences qu'ils peuvent aussitôt mettre en pratique dans leur vie quotidienne.

Etendre cette sensibilisation environnementale à d'autres écoles de campagne voire aux écoles urbaines - est vivement souhaitable. Dans cette perspective, gagner le soutien de l'Education Nationale pour l'introduction du thème Ecole Verte dans le programme scolaire et associer d'autres partenaires-supporteurs restent les défis majeurs posés à Sekoly Maitso. Il peut paraître effectivement secondaire pour le ministère malgache de l'Education de devoir, à l'échelle nationale, élaborer tout un programme sur l'environnement et former des enseignants compétents car une telle nouveauté demanderait des investissements publics supplémentaires et accentuerait une lourdeur administrative héritée déjà depuis des décennies. En outre, l'instabilité politique quasiment chronique dans le pays menace la continuité de projets novateurs.

\section{$\square$ Intégrer la dimension culturelle dans le développement rural}

Accorder une attention particulière à l'héritage culturel malgache figure parmi les principales approches de la Coopération suisse. Identifier les différents canaux artistiques particulièrement appréciés des Malgaches pour ensuite y faire passer le message environnemental s'est avéré comme un énorme pari. Pari, dans le sens qu'un très grand potentiel semblait s'offrir, mais que des obstacles importants restaient à surmonter. D'une part, parce que l'innovation apportée par un thème aussi vaste - et sérieux - que l'environnement dans les textes folkloriques pouvait déconcerter les adeptes de mpihira gasy, habitués à trouver détente et bonne humeur dans l'ironie des paroles de ce genre de chansons. Pour nouveau et important qu'il soit, ce thème semblait moins sujet à la raillerie que les sujets 
habituels. En somme, il s'agissait d'introduire des préoccupations de première importance sur des supports traditionnellement utilisés pour la pure distraction et cette orientation n'était pas acquise d'avance.

D'autre part, les spectateurs des mpihira gasy, depuis des générations, se sentaient proches des situations décrites pour avoir eux-mêmes vécu des sentiments comme l'amour, le mépris, la convoitise, etc. La dégradation de l'environnement est certes un phénomène beaucoup plus concret et palpable que les thèmes traditionnels cités ci-dessus : ses manifestations ne deviennent toutefois visibles et appréciables par les villageois que s'ils s'appliquent à regarder autour d'eux. Or, ces derniers ont généralement du mal - à cause de l'esprit colonial et de l'esprit socialiste qui l'a suivi - à «endosser » des problèmes considérés comme étant du domaine collectif. Le risque était donc que les villageois ne se sentent que faiblement impliqués par les descriptions véhiculées par les chansons et les considèrent comme une « relative faillite » par rapport à leur vocation de distraction.

En fait, les expériences des deux dernières années ont montré que ces craintes étaient injustifiées. Certaines troupes de mpihira gasy, particulièrement engagées pour la cause environnementale, ont su susciter l'intérêt et le plaisir des spectateurs. Elles sont parvenues à trouver l'équilibre entre des paroles très moralisatrices sur la dégradation de l'environnement et des airs suffisamment rythmés pour séduire les oreilles réticentes.

Afin d'étendre les destinataires de ces messages au public des villes, moins fanatique de mpihira gasy que les campagnards, la DDC a aussi fait appel à des chanteurs de variétés, tant suisses que malgaches, dans le cadre d'une campagne d'échanges culturels appelée Gasikara Maitso Volo (littéralement « Madagascar, Verte Contrée »). Ces artistes ont fait office de "messagers" dans le cadre de concerts dans les principales villes suisses et malgaches et, dans certains cas, leurs chansons sont devenues de véritables «tubes ». Gasikara Maitso Volo a permis de poser les bases d'un intéressant réseau de chanteurs et de médias sensibles à la cause verte. Toutefois, malgré la «percée » sur le terrain, cette voie culturelle n'a pas eu les suites désirées. Le problème était d'entretenir cette ouverture nouvelle des artistes afin que leur engagement n'en reste pas au stade embryonnaire et d'obtenir un soutien continu des pouvoirs publics. Or, les circonstances ont joué en défaveur de cette campagne malgré un écho largement positif dans les médias suisses et malgaches. D'une part, ne voyant cette campagne que sous son angle musical, certaines critiques se sont fait entendre, remettant en cause son bien-fondé et la justification de son coût. Du coup, dans un contexte financier difficile, l'engagement des pouvoirs publics malgaches s'est trouvé freiné. D'autre part, la nécessité de mettre en place un suivi de la campagne a coïncidé, à Madagascar, avec une période d'instabilité du gouvernement et force est de constater que quatre ministres successifs de la culture en sept mois n'ont pas réussi à défendre une approche novatrice mais peu évidente à mettre en place. Enfin, tenus par une obligation de résultats, certains responsables se sentent parfois « rattrapés par le temps » et sont rapidement déstabilisés dès que ces résultats se font attendre. Or, c'est justement dans le domaine du « sensible » que la coopération au développement demande du temps... Qui eut pu croire, après une saison de chansons, que les feux de brousse auraient déjà tous disparu? 


\section{$\square$ Informer le public et les décideurs sur les enjeux environnementaux}

Un des sujets les plus épineux à traiter avec les paysans malgaches est celui de l'utilisation de pesticides. Désaccoutumés de leurs pratiques naturelles ancestrales par une « culture DDT » qui remonte au tout début des années 70, les agriculteurs ont massivement introduit les produits chimiques à base de DDT dans leur exploitation. Par la suite, tout autre insecticide interdit d'utilisation dans les pays développés pour cause de toxicité et gracieusement offert par ceux-ci dans le cadre d'une aide bilatérale a été favorablement accueilli par le même public paysan via les commerçants et les dépôts communautaires de produits chimiques et pharmaceutiques.

Informer utilisateurs de pesticides et autorités des risques que comportent ces substances chimiques pour l'homme et son environnement apparaît donc comme la dernière née des approches environnementales retenues par la DDC.

Son expérience de 12 années en matière de pesticides agricoles va l'amener, par le biais d'un projet nommé Voarisoa (bonne création), à tenter une approche peu classique consistant à mettre les uns en garde contre les abus chimiques et à conscientiser les autres sur les « bonnes pratiques » en matière de produits dangereux (législation, formation, etc.). Mettant d'emblée les bénéficiaires du projet en contact direct avec les décideurs, Voarisoa ne fait rien par lui-même et entend jouer un rôle de forum permettant à des partenaires potentiels de se rencontrer en dehors des circuits formels. D'ailleurs, Voarisoa ne représente qu'un expert permanent, un secrétariat de deux personnes et un cortège de consultants nationaux intéressés et mobilisables selon la demande. Par exemple, Voarisoa a permis aux agents de la Santé Publique de s'appuyer sur des spécialistes en pesticides de l'agriculture pour améliorer leur formation en matière de traitements intra-domiciliaires contre les moustiques transmettant la malaria. De même, des producteurs de légumes ont pu avoir accès aux dernières « trouvailles » des chercheurs pour pouvoir entretenir leurs cultures suivant des méthodes écologiques et peu coûteuses. A un autre niveau, plus administratif, les agents de la Protection des Végétaux ont été soutenus dans leurs efforts pour procéder régulièrement à des contrôles de la qualité des pesticides sur le marché. Enfin, le comité malgache pour l'homologation des produits chimiques a été informé des derniers progrès en la matière, notamment auprès de la FAO et des conventions internationales concernées par ce sujet.

Un tel réseau d'apprentissage-information suscite énormément d'intérêt de la part des associations de producteurs et des distributeurs de pesticides, dès lors qu'ils ne se sentent pas liés de façon formelle au projet. Ne craignant pas de se trouver engagés dans une procédure administrative, ils peuvent répondre positivement aux visites de l'équipe de Voarisoa, voire solliciter à leur tour des séances d'information. Les « prestataires » de service mis en scène par Voarisoa, quant à eux, peuvent se féliciter de pouvoir répondre aux besoins d'information du public sans avoir à déployer des moyens d'action énormes et coûteux.

Dans cette ambiance prometteuse, il arrive cependant que l'on se heurte à des obstacles majeurs. D'abord, l'hostilité de quelques (re)vendeurs peu scrupuleux face aux contrôles de leurs marchandises, l'absence de législation claire en matière d'études d'impact pour l'implantation de nouvelles industries et - surtout - la faiblesse des pouvoirs publics pour vérifier le respect des réglementations en vigueur, sont autant de problèmes auxquels le projet est confronté. De 
plus, l'existence de plusieurs canaux d'importation et de distribution de pesticides complique la situation : ici, des succursales d'industries chimiques étrangères régies uniquement par des quotas annuels d'importation ; là, des « francstireurs » qui spéculent suivant la conjoncture ; en arrière plan, la libre circulation d'intrants chimiques non contrôlés provenant d'aides étrangères et au-dessus, les abus de certaines personnalités politiques en mal de profits.

Enfin, la réticence de quelques sociétés locales de reconditionnement de produits chimiques à faire publiquement état de leurs systèmes d'épuration et d'évacuation représente une véritable barrière à l'échange des informations. Et montre en outre qu'une telle coopération peut véritablement « déranger ».

Une ouverture à une meilleure communication entre importateurs/distributeurs et utilisateurs de pesticides est toutefois en voie d'être explorée : en milieu urbain, la presse peut jouer un rôle considérable dans l'information du grand public. En campagne, les associations de paysans peuvent constituer de véritables sources d'échanges d'expériences et d'apprentissage pour les nouvelles pratiques agricoles plus respectueuses de l'environnement.

\section{$\square$ Evaluer systématiquement le programme Madagascar-Suisse quant à son impact environnemental}

La Coopération malgacho-suisse perçoit à travers la variété d'approches dont elle dispose et que nous avons tenté d'illustrer jusqu'ici, d'autant de sources d'enseignement et d'outils pour ses actions qui ont clairement une visée environnementale. Au-delà de ces différentes approches et de leur champ d'action potentiel, la question de l'impact environnemental d'actions qui ont d'autres vocations (infrastructures rurales, santé, etc.) reste ouverte. Comment s'assurer que, pour l'ensemble du programme, chaque action soit bien consciente de son environnement et de l'influence qu'elle exerce sur lui ?

Pour tenter de répondre, au moins en partie, à cette question, la DDC a fait appel à l'Institut de Géographie de l'Université de Berne (Centre pour le développement et l'environnement) pour élaborer une grille d'indicateurs efficaces et adaptés. Sur la base de cet outil, une première action a été analysée en 1995 : un projet d'entretien routier dans la plus importante région rizicole malgache qui a permis aux riverains de limiter l'érosion et la déforestation, d'améliorer le système de drainage des routes et donc de limiter les glissements sur les bas-côtés, de prendre en charge eux-mêmes l'entretien régulier de la route, d'obtenir de l'ensemble des usagers le respect de la réglementation.

Lors d'un atelier-test ${ }^{9}$, équipe de projet, décideurs, opérateurs économiques, consultants et personnes extérieures se sont réunis pour chercher à mieux comprendre le comportement des différents groupes sociaux concernés par la vie de cet axe routier (villageois, riverains, transporteurs, usagers, etc.). Les échanges ont porté sur les différences de relation que chacun de ces groupes sociaux établit avec les composantes du projet et ont montré les implications des actions menées à différents niveaux. De tels échanges ont permis de mettre en lumière des situations de conflits potentiels entre ces différents groupes d'acteurs et/ou de bénéficiaires et les ont invités à les considérer sans les contourner. Cette

9 DDC Compte-rendu de l'atelier "Routes et Environnement », 1995, non publié, 38 p. 
manière de considérer une action non plus de manière isolée, mais en tentant d'en établir l'impact de manière plus élargie, présente de nombreux atouts, notamment en matière de prise de conscience des différents acteurs. Elle a en outre le mérite de mettre le doigt sur les principaux conflits d'intérêts. Ces derniers ne sont bien sûr pas résolus pour autant, mais une prise de conscience aussi objective que possible de la part des principaux acteurs concernés est déjà un pas dans la bonne direction.

Tentons d'illustrer en quels termes de tels conflits d'intérêts peuvent se présenter, à l'exemple de l'entretien de cet axe routier économiquement important. D'un côté, les villageois profitent de la meilleure accessibilité de la route et donc de l'augmentation du trafic, de la quantité de marchandises transportées et de prix plus avantageux. Ils étalent leurs marchandises en bordure de la voie et commencent à s'organiser pour maintenir le bon état de cette route à l'aide de barrières de pluie. De l'autre côté, de puissants transporteurs et marchands de riz et de charbon de bois souffrent de ne plus pouvoir prétexter du mauvais état de la route pour spéculer sur les prix de ces denrées essentielles. Ils chercheront donc par tous les moyens à décourager responsables et exécutants d'un tel projet. Rappelons que le principal expert suisse de ce programme a été assassiné en juillet dernier. Nous ne pouvons qu'espérer que ce drame n'ait été en rien lié à l'engagement de la DDC dans ce secteur. En attendant les résultats de l'enquête, les différents responsables sont néanmoins inquiets.

Refermons la parenthèse sur le cas de ce projet d'entretien routier. L'approche suivie s'est avérée pertinente pour mieux comprendre le contexte de l'action menée et la complexité des relations entre elle et son environnement. La DDC a décidé de retenir cette démarche, en la systématisant. Lors de chaque étape d'évaluation, d'orientation ou de planification, ses agents mèneront une telle analyse et apporteront des rectifications ou aménagements dans le cours des actions. De même, des indicateurs seront retenus et le suivi de leur évolution leur permettra de prendre en temps opportun les mesures adéquates.

Il reste que ce processus de suivi et d'évaluation systématique des impacts environnementaux est un processus exigeant en temps et en engagement. Il n'est pas toujours facile de mobiliser au juste moment les personnes requises. Il est aussi difficile d'obtenir des différents groupes d'acteurs qu'ils soient attentifs à la problématique environnementale et qu'ils décident de donner priorité la recherche de solutions spécifiques à cette problématique.

Par ailleurs, s'il faut les étendre à tout le programme de coopération entre Madagascar et la Suisse, de telles analyses d'impacts demandent à être adaptées aux différents contextes et aux divers niveaux de maturité des actions. Chacune d'entre elles étant spécifique, ainsi que les intervenants et les intérêts en jeu, les réponses à apporter aux problématiques sont peu prévisibles et devront encore être élaborées.

Par conséquent, là encore, les instruments d'évaluation ne pourront prouver leur efficacité qu'en fonction du temps qui aura été donné à leur utilisation. 


\section{BILAN GLOBAL}

Quel bilan tirer alors de cette diversité d'approches au service de l'environnement et comment la DDC évalue-t-elle l'ensemble des résultats obtenus au sein du programme de développement à Madagascar ?

Les personnes en charge du programme sont convaincues que la démarche poursuivie par la DDC à Madagascar s'inspire avantageusement des principes du développement durable. En clair, la Coopération suisse encourage, de par ses actions, les forces rurales actuelles à céder à la génération de demain un patrimoine mis en valeur de façon durable et protégé de la surexploitation. Selon elle, un tel engagement s'est soldé concrètement par un certain nombre de succès entrecoupés de quelques points négatifs qu'elle tente de transformer en défis à relever. Récapitulons-les.

Tout d'abord, l'approche participative défendue par la DDC a incité développeurs et paysans à se regrouper plus spontanément dans le but de réunir les outils nécessaires à la résolution des problèmes agricoles. En effet, plus les coopérations se rapprochent des villageois et respectent leurs traditions, plus elles gagnent la sympathie de ces derniers et plus les résultats tendent vers une responsabilisation progressive des paysans.

En outre, l'introduction de la notion d'environnement dans les actions a ouvert le champ à une collaboration plus étroite et soutenue entre agents de coopération et population locale. Elle a amené les uns à mettre en œuvre différentes approches en faveur de l'environnement, et a permis aux autres, via les appuis techniques, méthodologiques et structurels, d'améliorer leur maîtrise des questions environnementales et la gestion de leurs ressources naturelles vers plus de durabilité.

Enfin, le fait qu'au sein d'une population très majoritairement jeune, la DDC tente d'impliquer les enfants dès la première année de leur scolarité, donne des chances à cette population de voir s'ancrer progressivement, de génération en génération, les réflexes environnementaux.

Quoique insuffisants, ces trois résultats constituent des conditions de départ nécessaires pour un développement durable car, ensemble, jeunes et paysans devront trouver l'énergie nécessaire pour mobiliser les décideurs dans le sens d'une gestion écologique du sol, de la mer et de la forêt. Il reste néanmoins que pour pérenniser d'aussi importants acquis, cette coopération malgacho-suisse doit surmonter de nombreux obstacles, d'ordres structurel, politique et stratégique.

Premièrement, instaurer ce climat et cet état d'esprit respectueux de l'environnement suppose que les autorités locales définissent une stratégie claire quant à l'exploitation des ressources naturelles du pays. Or, il est souvent difficile pour un pays dont le PNB dépasse à peine les 200 US\$ par habitant de défendre une politique en faveur de l'environnement tout en encourageant les cultures d'exportation, exigeantes en surface et en intrants, mais génératrices de devises et de profits à court terme. Le défi est donc de tenir compte de ce conflit d'intérêts entre d'une part, les villageois qui (sur)vivent au jour le jour mais qui sont en mesure de mener avec assiduité des actions environnementales et d'autre part, les autorités publiques - à tous les niveaux - partagées entre la nécessité d'équilibrer une balance commerciale depuis longtemps déficitaire en exportant à outrance et 
de normaliser l'exploitation des sols, des forêts et de la mer grâce à l'aide financière des coopérations multilatérales. La coopération internationale a certainement un rôle à jouer à ce niveau en contribuant à jeter les bases d'une réflexion réglementaire amenant les pouvoirs publics à élaborer une loi pour le respect de l'environnement - à tous les échelons de la hiérarchie.

Un deuxième obstacle est d'ordre foncier. Les paysans seraient certainement plus motivés à consacrer leur temps et leur énergie à la protection des bases de leur existence s'ils étaient propriétaires de leurs champs plutôt que simples exploitants agricoles. Mais on touche là à un ensemble de privilèges très importants et de sérieux progrès seront encore nécessaires pour que l'accès à la terre soit garanti pour tous. Dans le cas de Madagascar, un mouvement assez large mais encore assez timide se fait dans ce sens, et il convient que tous ceux (ONG, agences de coopération, secteur privé) qui peuvent le soutenir coordonnent leurs efforts. Espérons que cette démarche induira une amélioration sensible de la situation agraire dans le pays avant que la spirale de la pauvreté et de la dégradation n'entraîne de trop fortes tensions sociales.

Enfin, dans le contexte encore timide du dialogue entre les nations pour la sauvegarde des écosystèmes menacés, chacun gagnerait à échanger régulièrement ses expériences sur la tribune internationale, écouter ses voisins et présenter le bilan des expériences faites - y compris des bilans d'expérience de coopération - afin d'entretenir cette dynamique capitale... : pour un nouvel ordre environnemental mondial. Une utopie ?

\section{Glossaire des termes en malgache}

\begin{tabular}{ll}
\hline Gasikara Maitso Volo : slogan créé par le ministère malgache de la Culture en mai 1995 pour \\
baptiser le projet de sensibilisation culturelle et environnementale mis \\
en œuvre par ledit ministère et la coopération suisse. Il évoque un \\
Madagascar de verdure
\end{tabular}

